\title{
Raisonnement clinique et stratégies d'intervention : entrevue simulée auprès de mères consommatrices de substances psychoactives suivies en protection de la jeunesse Clinical reasoning and intervention strategies: simulated interviews with psychoactive substance-using mothers who monitored by youth protection services
}

\author{
Sophie Couture, Myriam Laventure, Annick Bourget, Clémence Pentecote et \\ Fannie Fafard
}

Volume 47, numéro 2, 2018

URI : https://id.erudit.org/iderudit/1054058ar

DOI : https://doi.org/10.7202/1054058ar

Aller au sommaire du numéro

Éditeur(s)

Revue de Psychoéducation

ISSN

1713-1782 (imprimé)

2371-6053 (numérique)

Découvrir la revue

Citer cet article

Couture, S., Laventure, M., Bourget, A., Pentecote, C. \& Fafard, F. (2018).

Raisonnement clinique et stratégies d'intervention : entrevue simulée auprès de mères consommatrices de substances psychoactives suivies en protection de la jeunesse. Revue de psychoéducation, 47(2), 213-230.

https://doi.org/10.7202/1054058ar

\section{Résumé de l'article}

Les professionnels intervenant auprès de parents présentant un double problème de négligence envers leurs enfants et de consommation problématique de substances psychoactives sont confrontés quotidiennement à des prises de décisions complexes. Avant de statuer sur les meilleures pratiques à préconiser dans le domaine, il semble important de mieux comprendre les types de raisonnement clinique et les stratégies d'intervention utilisés par les intervenants pour résoudre un problème et prendre des décisions. Une recherche a donc été menée afin d'identifier les types de raisonnement clinique (pragmatique, procédural, interactif et conditionnel) et les stratégies d'intervention auxquels les intervenants travaillant auprès de cette clientèle ont le plus souvent recours et d'illustrer les regroupements entre ces raisonnements et les stratégies d'intervention associées. Pour ce faire, l'utilisation de l'entretien d'explicitation (Vermersch, 2006) a permis d'accéder au savoir implicite de huit intervenants rencontrés après une entrevue clinique simulée avec une mère-comédienne. Bien que l'ensemble des types de raisonnement se retrouve dans la pratique des intervenants, le raisonnement interactif prédomine et s'observe particulièrement lors des stratégies d'évaluation clinique (par exemple, évaluer la reconnaissance des faits) et relationnelle (par exemple, renforcer la confiance des parents). Une meilleure compréhension des raisonnements cliniques et des stratégies d'intervention utilisées lors de prises de décisions cliniques auprès de parents négligents et consommateurs de substances psychoactives représente une première étape pour améliorer le soutien offert aux intervenants et aux gestionnaires en matière de formation et de supervision clinique.
Tous droits réservés @ La Corporation de la Revue Canadienne de Psycho-Éducation, 2018
Ce document est protégé par la loi sur le droit d'auteur. L’utilisation des services d’Érudit (y compris la reproduction) est assujettie à sa politique d'utilisation que vous pouvez consulter en ligne.

https://apropos.erudit.org/fr/usagers/politique-dutilisation/ 


\section{Raisonnement clinique et stratégies d'intervention : entrevue simulée auprès de mères consommatrices de substances psychoactives suivies en protection de la jeunesse}

\section{Clinical reasoning and intervention strategies: simulated interviews with psychoactive substance-using mothers who monitored by youth protection services}

\section{S. Couture ${ }^{1}$}

M. Laventure ${ }^{2}$

A. Bourget ${ }^{3}$

C. Pentecote ${ }^{4}$

F. Fafard ${ }^{5}$

\section{Institut universitaire Jeunes en} difficulté, CIUSSS du Centre-

Sud-de-l'île-de-Montréal;

École de criminologie,

Université de Montréal

2 Département de

psychoéducation, Université de

Sherbrooke

3 École de réadaptation, Université de Sherbrooke

4 Institut universitaire Jeunes en difficulté, CIUSSS du Centre-

Sud-de-l'île-de-Montréal

5 Direction du programme santé mentale et dépendance, CIUSSS de la Montérégie-

Ouest; Institut universitaire sur les dépendances, CIUSSS du

Centre-Sud-de-l'île-de-Montréal

\section{Correspondance :}

Sophie Couture

IUJD, CIUSSS du Centre-Sudde-l'île-de-Montréal

1001 boul. de Maisonneuve est Montréal (Québec) H2L 4R5, $7^{\mathrm{e}}$ étage

Tél.: 514-896-3485

Téléc. : 514-896-3400

sophie.couture.ccsmtl@ssss.

gouv.qc.ca
Résumé

Les professionnels intervenant auprès de parents présentant un double problème de négligence envers leurs enfants et de consommation problématique de substances psychoactives sont confrontés quotidiennement à des prises de décisions complexes. Avant de statuer sur les meilleures pratiques à préconiser dans le domaine, il semble important de mieux comprendre les types de raisonnement clinique et les stratégies d'intervention utilisés par les intervenants pour résoudre un problème et prendre des décisions. Une recherche a donc été menée afin d'identifier les types de raisonnement clinique (pragmatique, procédural, interactif et conditionnel) et les stratégies d'intervention auxquels les intervenants travaillant auprès de cette clientèle ont le plus souvent recours et d'illustrer les regroupements entre ces raisonnements et les stratégies d'intervention associées. Pour ce faire, l'utilisation de l'entretien d'explicitation (Vermersch, 2006) a permis d'accéder au savoir implicite de huit intervenants rencontrés après une entrevue clinique simulée avec une mère-comédienne. Bien que l'ensemble des types de raisonnement se retrouve dans la pratique des intervenants, le raisonnement interactif prédomine et s'observe particulièrement lors des stratégies d'évaluation clinique (par exemple, évaluer la reconnaissance des faits) et relationnelle (par exemple, renforcer la confiance des parents). Une meilleure compréhension des raisonnements cliniques et des stratégies d'intervention utilisées lors de prises de décisions cliniques auprès de parents négligents et consommateurs de substances psychoactives représente une première étape pour améliorer le soutien offert aux intervenants et aux gestionnaires en matière de formation et de supervision clinique.

Mots-clés : intervention, raisonnement clinique, négligence, consommation problématique de substances psychoactives, entretien d'explicitation 


\begin{abstract}
Professionals who work with parents presenting a double problem of negligence towards their children and of problematic psychoactive substance use are confronted with complex decision-making on a daily basis. Before determining what best practices are recommended in this field, it seems important to better understand the various types of clinical reasoning and intervention strategies used by professionals to resolve issues and make decisions. Therefore, a research project was conducted to identify the types of clinical reasoning (pragmatic, procedural, interactive and conditional) and the response strategies most often used by counselors working alongside this clientele, as well as to illustrate the clustering between these reasoning processes and the response strategies associated to them. To that end, the use of the explicitation interviewing technique (Vermersch, 2006) allowed us to access the implicit knowledge of eight counselors, who were met after a simulated clinical interview with an actress playing a mother. Although all types of reasoning processes are found in the practice of counselors, interactive reasoning predominates and is particularly present in the context of clinical evaluation (for example, when evaluating fact recognition) and relational (for example, when reinforcing the confidence of parents) strategies. A better understanding of clinical reasoning processes and of intervention strategies used during clinical decision-making among negligent, psychoactive substance users, represents a first step in improving the support offered to counselors and managers in terms of training and clinical supervision.
\end{abstract}

Keywords: intervention, clinical reasoning, negligence, problematic psychoactive substance use, explicitation interviewing

\title{
Introduction
}

Au Québec, la présence d'une consommation problématique d'alcool ou de drogues ${ }^{1}$ peut être associée aux signalements évalués en protection de la jeunesse. En effet, une consommation problématique d'alcool connue ou soupçonnée s'observe chez au moins un parent dans 13,6 \% des cas évalués en protection de la jeunesse et la présence d'une consommation problématique aux drogues connue ou soupçonnée s'observe dans 17,9 \% des cas évalués (Hélie, Collin-Vézina, Trocmé, Turcotte et Girouard, 2017). Considérant les divers problèmes vécus (par exemple absence de soutien social, trouble de santé mentale; Barnard, 2007; Conners et al., 2004; Mayes et Truman, 2002) par ces parents présentant un double problème de négligence envers leurs enfants et de consommation problématique d'alcool et de drogues, les professionnels intervenant auprès de cette clientèle doivent souvent conjuguer avec un portrait clinique complexe.

Depuis une vingtaine d'années, le programme montréalais « Jessie, ensemble pour protéger les tout-petits ॥ (ci-après désigné : programme Jessie) est offert en partenariat entre les services de protection de l'enfance et de la jeunesse et les services de réadaptation en dépendance du Centre intégré universitaire de

1. Une consommation problématique se définit par un mode d'utilisation inapproprié d'alcool ou de drogues, entrainant une détresse ou un dysfonctionnement dans différentes sphères de vie de la personne (American Psychiatric Association, 2013). 
santé et de services sociaux du Centre-Sud-de-l'Île-de-Montréal (CCSMTL) ${ }^{2}$. Ce programme s'adresse aux parents dont la consommation d'alcool, de drogue ou de jeu entraîne des répercussions sur la sécurité ou le développement de leurs enfants de $0-5$ ans (ci-après désigné : parents négligents et consommateurs). Les intervenants du programme Jessie travaillent selon les préceptes de l'approche de réduction des méfaits et de l'approche motivationnelle. Dans ce contexte, la réduction des méfaits vise à « mobiliser le parent dans la recherche et la mise en application de solutions permettant de réduire les méfaits de sa consommation pour assurer la continuité des soins et la stabilité des liens auxquelles l'enfant a droit et dont il a besoin pour bien se développer " (Comité de coordination du programme Jessie, 2014, p. 6). Puisque la participation au programme Jessie est volontaire et que le parent est considéré comme le principal responsable de son enfant, il importe de considérer la motivation du parent dans la démarche clinique (Comité de coordination du programme Jessie, 2014). Selon l'approche motivationnelle, une intervention visant la modification d'un comportement doit prendre en considération l'étape de changement à laquelle se trouve le parent (c'est-à-dire précontemplation, contemplation, préparation, action, maintien) afin d'assurer un changement durable (DiClemente et Velasquez, 2002). Les professionnels qui travaillent dans le cadre du programme Jessie doivent concilier des approches et des philosophies de travail différentes selon le service dans lequel il exerce. Les intervenants qui travaillent pour les services de réadaptation en dépendance ont pour client le parent, alors que les intervenants des services de protection de l'enfance et la jeunesse ont pour client l'enfant. Leur objectif commun est de mobiliser le parent tout en veillant et en garantissant la sécurité et le développement de l'enfant en vertu de la Loi sur la protection de la jeunesse.

\section{Le raisonnement clinique}

Pour bien concevoir les pratiques cliniques des intervenants du domaine de la protection de la jeunesse, certains auteurs se sont attardés à la compréhension de leur prise de décisions. Parmi les modèles dominants, le cadre théorique du Decision-making ecology (DME) permet la distinction des différents facteurs impliqués dans la prise de décisions en contexte de protection de la jeunesse (Baumann, Dalgleish, Fluke et Kern, 2011; Fluke, Baumann, Dalgleish et Kern, 2014; Shlonsky, 2015). L'un des aspects centraux du DME est l'intérêt porté à l'intervenant pour accéder au processus de prise de décisions. Ces aspects concernent entre autres éléments les processus psychologiques qui permettent aux intervenants de réfléchir à la situation et de "passer à l'action ». En lien avec le modèle DME, la présente recherche propose une application empirique spécifique sous l'angle du raisonnement clinique. Elle s'inspire de divers domaines des sciences de la santé (par exemple médecine, optométrie, ergothérapie) ayant développé au cours des dernières décennies une expertise en matière de raisonnement clinique (Higgs et Jones, 2008).

2. Anciennement, ces instituts se nommaient le Centre jeunesse de Montréal-Institut universitaire et le Centre de réadaptation de dépendance-Institut universitaire. Depuis le 1er avril 2015, ces instituts ont intégré le Centre intégré universitaire de santé et de services sociaux du Centre-Sud-de-l'lle-de-Montréal. 
Le raisonnement clinique peut se définir comme un processus cognitif permettant à l'intervenant de gérer les situations cliniques complexes qui lui sont présentées (Nendaz, Charlin, Leblanc et Bordage, 2005; Newble, Norman et van der Vleuten, 2000; Richard, 2008). Ce processus cognitif représente une « activité intellectuelle par laquelle le clinicien synthétise l'information obtenue dans une situation clinique, l'intègre avec les connaissances et les expériences antérieures et les utilise pour prendre des décisions de diagnostic et de prise en charge " (Nendaz et al., 2005, p. 236). Le raisonnement clinique est de nature complexe en raison de la tâche effectuée et des enjeux associés à chaque milieu (par exemple considérer les priorités de tous les partis, prendre des décisions; Higgs et Jones, 2008; Andrews et Syeda, 2017).

Le raisonnement clinique peut se décliner selon quatre types complémentaires : le raisonnement pragmatique, procédural, interactif et conditionnel. Ces types de raisonnement permettent lors de l'analyse réflexive de l'intervention d'identifier quel(s) type(s) de raisonnement est utilisé par l'intervenant (Fleming, 1991; Schell et Cervero, 1993; Unsworth, 2004). D'abord, le raisonnement pragmatique tient compte de tout ce qui entoure la situation d'évaluation. L'intervenant considère alors l'environnement de travail, les connaissances et les aptitudes personnelles de même que les valeurs et les ressources financières du client. Le raisonnement pragmatique inclut également les références personnelles et professionnelles des intervenants eux-mêmes (par exemple répertoire d'habiletés, valeurs) (Schell et Cervero, 1993). Le raisonnement procédural met l'accent sur les incapacités de la personne et sur les façons de les régler. Ce type de raisonnement est associé aux stratégies d'intervention visant l'amélioration des capacités de la personne. Ce type de raisonnement s'observe lorsque l'intervenant aborde les différentes séquences de l'intervention soit le diagnostic, la prescription d'objectifs et le pronostic. Ensuite, le raisonnement interactif s'attarde au point de vue de la personne plutôt qu'à ses difficultés. II met l'accent sur les difficultés, mais du point de vue du client. Ce type de raisonnement se solde souvent par l'élaboration conjointe de buts et de stratégies. Enfin, le raisonnement conditionnel apparait habituellement plus tard au cours du suivi. L'intervenant peut alors anticiper, à partir de ses expériences passées, l'amélioration ou la dégradation de l'état du client dans le futur, le pronostic. C'est le bagage d'expériences auquel se réfère l'intervenant qui lui permet d'anticiper les possibilités de succès et d'échecs (Fleming, 1991; Schell et Cervero, 1993; Unsworth, 2004).

Bien qu'il soit reconnu que la prise de décisions soit centrale à la pratique (Taylor, 2006), aucune étude répertoriée ne s'est spécifiquement attardée aux types de raisonnement clinique et aux stratégies d'intervention employés par les intervenants œuvrant auprès de parents négligents et consommateurs de substances psychoactives. Or, l'identification et la description des stratégies d'intervention et des types de raisonnement des intervenants représentent une première étape essentielle à l'amélioration des pratiques auprès de cette clientèle. En effet, avant de statuer sur les meilleures pratiques à prioriser, il importe de détailler la pratique clinique telle que vécue par les intervenants. Pour accéder à ce savoir tacite, la présente étude vise à accompagner l'intervenant dans une pratique réflexive telle que proposée dans l'entretien d'explicitation de Vermersch (2006; Guillemette, 2016). Un tel entretien permettra d'accéder au discours intérieur et 
d'identifier les types de raisonnement clinique et les stratégies d'intervention utilisés par les intervenants.

\section{Objectif}

La présente étude vise à (1) identifier les types de raisonnement clinique (pragmatique, procédural, interactif et conditionnel) et les stratégies d'intervention utilisés par les intervenants du programme Jessie à l'aide d'une entrevue clinique simulée et (2) illustrer les regroupements entre les types de raisonnement clinique et les stratégies d'intervention.

\section{Méthode}

La présente étude utilise un devis qualitatif qui s'inspire de la recherche descriptive interprétative (Gallagher, 2014). L'étude a été acceptée par le Comité d'éthique Jeunes en difficulté, le Comité d'éthique de la recherche en toxicomanie et le Comité d'éthique de la recherche - Éducation et sciences sociales de l'Université de Sherbrooke.

\section{Participants}

Le recrutement des participants s'est déroulé parmi les 28 intervenants du programme Jessie. Plus spécifiquement, les intervenants devaient avoir travaillé un minimum de trois jours par semaine dans un service de protection de l'enfance et de la jeunesse ou un service de réadaptation en dépendance depuis plus de trois ans. La vidéo étant essentielle pour recueillir des données rigoureuses et valides dans le cadre de ce projet, les intervenants refusant d'être filmés et enregistrés ont été exclus.

Pour décrire l'échantillon, les intervenants $(n=8)$ ont répondu à un questionnaire maison portant sur leurs caractéristiques sociodémographiques et leurs expériences cliniques. Parmi les huit intervenants de la présente étude, on retrouve six femmes. Les intervenants composant l'échantillon sont âgés en moyenne de 40,5 ans (27-60 ans; É.-T. = 12,06), travaillent tous à temps plein dans le domaine de l'intervention en moyenne depuis 16 ans (3-32 ans; É.-T. = 9,91). De plus, sept intervenants ont minimalement un diplôme de niveau baccalauréat. Les domaines de formations des intervenants sont en travail social $(n=2)$, psychoéducation $(n=$ 2 ), criminologie $(n=2$ ) et sciences sociales (cumul de diplômes, $n=2$ ).

Bien que cet échantillon soit limité, les huit intervenants rencontrés ont permis d'atteindre une saturation empirique des données (c'est-à-dire que les dernières entrevues n'apportaient plus d'informations nouvelles). Pour s'assurer que cet échantillon de convenance soit également représentatif de la population des intervenants du programme Jessie, une comparaison a été effectuée entre les participants de la présente étude $(n=8)$ et d'autres intervenants du programme ayant participé à une précédente étude $(n=14)$ (Laventure, Couture, Fafard et Pentecôte, 2016). Les participants ne se distinguent pas en ce qui concerne leur sexe, leur âge, leurs années en intervention ou leur formation en toxicomanie (données non présentées). 


\section{Procédures}

Pour accéder au raisonnement clinique des intervenants du domaine de la protection de la jeunesse et de la dépendance, les chercheuses ont soumis les intervenants à un entretien d'explicitation. La procédure de collecte de données s'inspire d'études ayant exploré le raisonnement clinique d'étudiants en médecine (Bourget, 2013), d'optométristes (Faucher, 2012), d'ergothérapeutes (Carrier, Levasseur, Bédard et Desrosiers, 2012), d'intervenants en soutien à domicile (Ruest, Bourget, Delli-Colli et Guay, 2017) ainsi que des infirmiers anesthésistes diplômés d'état (Deroche, Girard, Naudin, Bourget, 2016).

\section{Entretien d'explicitation}

Entrevue clinique simulée. Pour effectuer l'entretien d'explicitation, une entrevue clinique simulée a d'abord été organisée afin de recréer le contexte et des situations cliniques habituellement rencontrées par les intervenants. Pour assurer une représentativité des situations cliniques habituellement rencontrées par les intervenants, deux vignettes cliniques (vignette 1: Julie; vignette 2: Geneviève) ont été élaborées par une intervenante et une gestionnaire spécialisées dans l'intervention auprès de cette clientèle. Deux comédiennes professionnelles rémunérées ont été attitrées à l'une ou l'autre de ces vignettes en plus de participer à une rencontre préparatoire avec un membre de l'équipe de recherche. Lors de cette rencontre, les comédiennes ont été informées de la situation de la mèrecomédienne (c'est-à-dire situation familiale et sociale, parcours scolaire, détails du signalement, relation avec le conjoint), l'attitude à privilégier (par exemple répondre aux questions de façon prompte et de mauvaise humeur, avoir l'air abattue et dépassée par les évènements) et de l'importance d'uniformiser leur jeu pour l'ensemble des intervenants. Ainsi, la moitié des intervenants ont dû intervenir comme ils le font habituellement auprès de la mère-comédienne nommée Julie et l'autre moitié, auprès de la mère-comédienne Geneviève.

La journée précédant l'entrevue clinique simulée, les intervenants ont reçu par courriel la vignette clinique énumérant les grandes lignes de la situation de la mère-comédienne. Chaque intervenant a reçu comme unique consigne de prendre connaissance de ces éléments pour réaliser une entrevue, tel qu'ils le font habituellement lors d'un premier contact avec un parent suivi dans le cadre du programme Jessie. Les intervenants étaient donc libres d'utiliser les stratégies d'intervention de leur choix avec la mère-comédienne.

Le jour de la rencontre, chaque intervenant était rencontré individuellement pour lire et signer le formulaire de consentement de participation à la recherche. Puis, chaque intervenant disposait d'une quinzaine de minutes pour relire, au besoin, la vignette clinique. Ensuite, chaque intervenant réalisait l'entrevue clinique simulée, d'une durée approximative de 45 minutes avec la mère-comédienne. L'entrevue était enregistrée numériquement sur vidéo selon une perspective subjective située (Rix et Biache, 2004). La perspective subjective située permettait non pas de filmer l'intervenant, mais bien ce qu'il regardait, ce à quoi il portait attention tout au long de son entrevue. Pour obtenir cette perspective, la caméra vidéo était placée dans le même champ de vision que celui de l'intervenant. En conséquence, en se servant 
du visionnement de la vidéo, l'intervenant était replongé dans le même état cognitif dans lequel il était avec la mère-comédienne. Cet enregistrement en perspective située constituait le matériel à partir duquel se réalisait l'entretien d'explicitation. Afin de préparer cet entretien d'explicitation, une paire d'écouteurs était branchée dans la caméra vidéo permettant ainsi à la chercheuse d'écouter et de prendre des notes en simultanée dans une salle attenante.

Entretien d'explicitation. Après l'entrevue clinique simulée, une pause d'une quinzaine de minutes a été offerte aux intervenants. Par la suite, une chercheuse a rencontré l'intervenant pour procéder à l'entretien d'explicitation à l'aide de la vidéo. Pour effectuer ce type d'entretien, les chercheuses ont été formées aux principes de base de cette technique de pratique réflexive. Développé par Vermersch, l'entretien d'explicitation se caractérise par un « ensemble de pratiques d'écoute basées sur des grilles de repérage de ce qui est dit et de techniques de formulation de relances (questions, reformulations, silences) qui visent à aider, à accompagner la mise en mots d'un domaine particulier de l'expérience en relation avec des buts personnels et institutionnels divers » (Vermersch, 2006, p. 17).

Les entretiens d'explicitation ont duré en moyenne 90 minutes. À l'aide de la vidéo, l'entretien a visé à replacer l'intervenant dans son expérience, telle qu'il l'a vécue. La chercheuse a recueilli les verbalisations détaillées du déroulement des actions cognitives et des informations prises en compte lors de l'entrevue avec la mère-comédienne. Jumelé à la vidéo, l'entretien d'explicitation a permis de recréer l'expérience complète en demandant à l'intervenant d'expliquer chacune de ses interventions. Pour les besoins de la recherche, cet entretien a été enregistré sur support audio.

Validation. Peu de temps après l'entretien d'explicitation, les chercheuses ont effectué une synthèse de l'ensemble des stratégies d'intervention utilisées par l'intervenant lors de l'accueil, le déroulement et la clôture de l'entrevue clinique simulée. Cette synthèse a été effectuée à l'aide du contenu de l'enregistrement audio de l'entretien d'explicitation et des notes des chercheuses. Ainsi, une semaine après cet entretien, une entrevue semi-structurée individuelle d'une trentaine de minutes a été réalisée avec chacun des intervenants afin de valider la synthèse des stratégies d'intervention utilisées lors de l'entretien d'explicitation. Pendant la rencontre de validation, chaque intervenant a été invité à clarifier et à compléter les informations manquantes ou corriger les informations erronées. Cette rencontre a été enregistrée sur support audio.

\section{Analyse des données}

Pour répondre aux objectifs de l'étude, différents processus d'analyses de données ont été suivis (Gallagher, 2014; Miles et Huberman, 2005). Les analyses ont été effectuées à l'aide du logiciel NVivo 10. Pour le premier objectif de l'étude, soit identifier les types de raisonnement clinique et les stratégies d'intervention utilisés par les intervenants, la première série d'analyse a consisté en l'écoute et la lecture des notes de l'entretien d'explicitation. Selon le moment de l'entrevue (accueil, déroulement et clôture de l'entrevue), les chercheuses ont ensuite répertorié et schématisé en ordre chronologique les stratégies d'intervention mises 
de l'avant par les intervenants. Cette analyse a été complétée et validée avec tous les intervenants lors de la rencontre de validation. La deuxième série d'analyse a consisté en la condensation des stratégies d'intervention dans une grille d'analyse effectuée par deux membres de l'équipe de recherche. Après la transcription des verbatims de l'entretien d'explicitation, une troisième série d'analyses a été effectuée par les membres de l'équipe. Chaque extrait de verbatims ( $n$ total $=684$ extraits) a été codifié selon les quatre types de raisonnement clinique (raisonnement pragmatique, procédural, interactif et conditionnel) et les stratégies d'intervention répertoriées dans la grille d'analyse par un membre de l'équipe de recherche. Ensuite, cette codification a été corroborée par un autre membre de l'équipe. Les divergences de codification ont été reprises lors d'une discussion visant l'atteinte d'un consensus. Pour la présentation des quatre types de raisonnement clinique, le pourcentage d'extraits associés à chaque type de raisonnement est précisé. Également, chaque type de raisonnement utilisé est illustré à l'aide d'extraits de verbatim. En ce qui concerne l'identification des stratégies d'intervention, le résultat final de la classification et du regroupement des données est présenté.

Pour répondre au deuxième objectif de l'étude, soit illustrer les regroupements entre les types de raisonnement clinique et les stratégies d'intervention, les codifications (types de raisonnement et stratégies d'intervention) effectuées pour chaque extrait de verbatim ( $n$ total $=684$ extraits) ont été utilisées.

\section{Résultats}

\section{Types de raisonnement clinique utilisés par les intervenants}

À partir de la typologie de Fleming (1991), Schell et Cervero (1993) et Unsworth (2004), les dires des intervenants lors des entretiens d'explicitation ont été classés en quatre types de raisonnement différents : pragmatique, procédural, interactif et conditionnel. Tel qu'illustré dans la figure 1, parmi les extraits codifiés, $31,6 \%$ des propos étaient rattachés à un raisonnement de type pragmatique, $24,8 \%$ au type procédural, $36,1 \%$ au type interactif et $7,5 \%$ au type conditionnel.

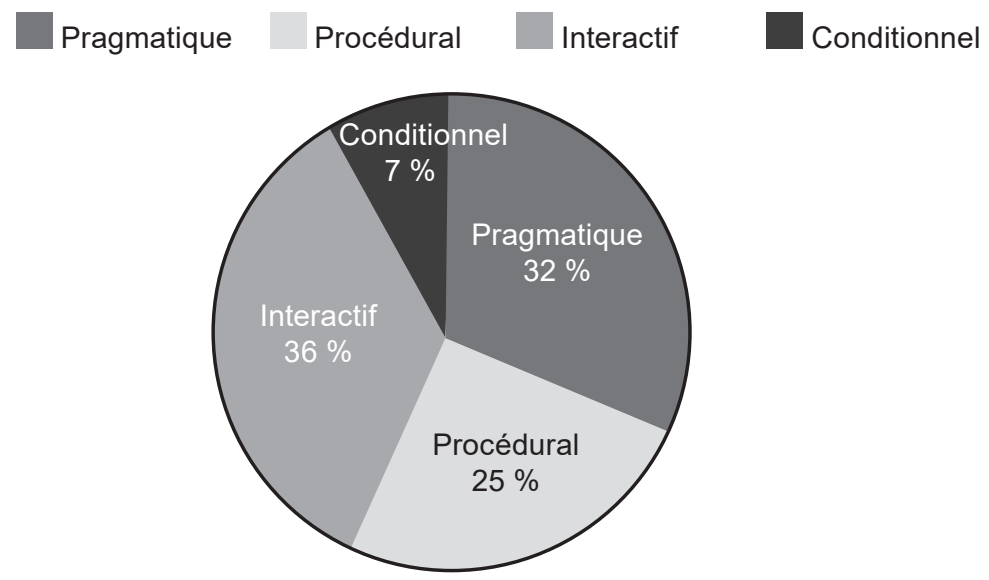

Figure 1. Raisonnement clinique des intervenants 
Le raisonnement pragmatique était axé sur le contexte de l'individu, mais aussi de l'intervenant. Tel qu'observé (voir figure 1), ce type de raisonnement est fréquemment utilisé par les intervenants. Comme son nom l'indique, les intervenants faisaient appel au raisonnement pragmatique pour expliquer le déroulement " logique » et " attendu » d'une première rencontre d'évaluation. En fait, les intervenants se servaient surtout de ce type de raisonnement pour évaluer et recueillir le point de vue du client par rapport à sa situation et pour justifier les prochaines étapes d'évaluation au cours de leur entrevue. Dans l'extrait suivant, l'intervenant considérait le contexte social et économique du parent pour planifier son intervention :

"Le fait qu'ils soient inscrits à la garderie me permet de savoir comment les enfants arrivent le matin et dans quel état les parents viennent le chercher. On sait que lorsque les enfants sont à la garderie, ils sont 40 heures par semaine dans un endroit adéquat. On aime ça quand ils sont à la garderie. Elle est un peu jeune par exemple, elle a 3-4 mois. Je voulais voir pour les prochains mois c'était quoi son intérêt mais aussi à Hochelaga il est possible de faire des demandes en avance pour avoir des places pour les enfants » (participant 1).

Le raisonnement pragmatique s'observait également dans la capacité de l'intervenant à considérer ses habiletés personnelles :

"C'est important que les gens sentent qu'on est une équipe et qu'on se fait confiance les uns les autres. Moi je ne me sens pas habileté à aller parler de toxicomanie avec les parents, non, j'ai mes limites. Je sais que si elle me parle de quelque chose, je sais la valeur que ça a, mais je ne suis pas nécessairement la bonne personne pour le traiter avec elle » (participant 2).

Dans le cadre d'une première rencontre avec un parent, les intervenants se servaient aussi du raisonnement procédural pour expliquer les diverses séquences de l'intervention clinique. Ainsi, ce raisonnement leur permettait de mettre en place des actions concrètes nécessaires pour établir un diagnostic clinique, un pronostic à plus long terme et offrir les services adaptés à la situation de la famille.

"Comme des hypothèses à savoir si elle veut vraiment être avec son conjoint ou ce qu'elle veut c'est ne pas être toute seule. Par hypothèse, je veux dire que je vais aller vérifier par une question» (participant 6).

"Ça fait que je regarde dans les prochaines semaines, pis ça ne peut pas prendre éternellement de temps non plus. Le speed il faut qu'elle s'arrête, faut qu'elle sorte de la maison, pas le choix. Ça c'est l'information que je donne à la DPJ, qu'est-ce qu'on fait? Les deux consomment, donc on fait quoi? On sort les enfants de là? On les met en famille d'accueil? Y'as-tu de la famille qui peut les prendre? Y'as-tu la grand-mère de Thomas qui peut l'aider pendant 10 jours, le temps qu'on l'arrête et qu'on la fasse voir par le médecin pis qu'on voit si..., il y a tout la partie psychiatrique qu'il va falloir regarder. Cette femme-là es-tu en détresse psychologique, en dépression majeure? Je sais pas encore » (participant 8). 
Le raisonnement interactif, le plus utilisé par les intervenants dans la présente étude, provenait de l'élaboration conjointe avec le parent de buts et de stratégies à mettre de l'avant lors de l'intervention. Dans ce type de raisonnement, l'intervenant utilisait différentes stratégies relationnelles afin d'encourager et de maximiser la participation du parent. Dans le cadre de la première rencontre, les intervenants y ont eu recours pour considérer le " ressenti », l'aspect davantage émotif de l'évaluation du parent. Cette lecture relationnelle lors de l'entrevue permettait à l'intervenant d'adapter ses attitudes interpersonnelles afin d'établir et de maintenir la relation avec le parent :

" Je regarde son non verbal. Par exemple, si je parle de la situation de compromission et qu'elle se met à soupirer ça va être plus difficile alors on va y aller avec des pincettes. Voir comment son corps réagi me donne un indice de ce qui va être plus difficile à aborder ou pas » (participant 6).

"Quand elle a pleuré tantôt, je l'ai sentie vulnérable, pas méfiante. Si j'avais senti de la méfiance, je l'aurais nommé tout de suite. Elle, elle est plus vulnérable que méfiante. Ouais, en fait, il va plus falloir s'en occuper comme il faut pour ne pas lui faire peur. Si quelqu'un avait été méfiant, ça c'est tout le temps des affaires que je nomme en première rencontre pour essayer de défaire ça afin de ne pas partir sur des fausses idées » (participant 2).

" Je vois qu'elle est en maudit que la PJ [protection de la jeunesse] soit dans sa vie. Je veux savoir pourquoi elle est en maudit, c'est quoi sa perception de la situation. Est-elle en maudit parce qu'elle s'est fait pogner, parce qu'elle était encore gelée, parce que son conjoint n'a rien foutu, fâché par rapport à elle-même parce qu'elle a confié ses enfants à son conjoint? Je veux voir sa perception pour pouvoir partir de là au niveau du problème ॥ (participant 3).

Tel qu'illustré dans l'analyse des verbatims des entretiens d'explicitation, le raisonnement conditionnel a été celui le moins souvent rencontré lors d'une première rencontre avec le parent. L'intervenant utilisait un raisonnement conditionnel lorsqu'il prenait en considération l'expérience du client pour faire son pronostic. Bien que le raisonnement conditionnel s'observe généralement après les premières rencontres, il est tout de même possible de constater que les intervenants s'en servaient à l'occasion pour prédire l'amélioration ou la dégradation de la situation selon les interventions proposées.

"Parce que son fils ne doit pas être bien dans cette situation-là. À un moment donné le plus jeune, va grandir et s'en rendre compte de cette injustice. II pourrait profiter de cette situation. Je vois de futurs conflits familiaux encore plus grands, d'où l'importance d'intervenir bientôt làdessus » (participant 3).

"Oui, il y a quelques affaires, comme l'alcoolisme et la non-reconnaissance des impacts sur le bébé, mais elle s'est livrée quand même assez et est 
capable de faire des liens. Je crois que ça va être quelqu'un avec qui il sera agréable de travailler. Un bon pronostic, je pense » (participant 6).

Dans un processus d'évaluation clinique, force est de constater que les intervenants utilisaient les différents types de raisonnement clinique, et ce, dans des combinaisons variées. Ainsi, les intervenants pouvaient alterner les types de raisonnement prenant parfois une position davantage axée sur le contexte de l'évaluation (raisonnement pragmatique), sur l'histoire du parent (raisonnement interactif), sur les processus cliniques en cours (raisonnement procédural) et puis sur les stratégies relationnelles utilisées pour comprendre la situation du parent (raisonnement interactif).

«Au début, moi je fais souvent tester afin de voir si c'est vraiment volontaire. Des fois pour pas aller au tribunal, pour se débarrasser $d u$ papier ou de la situation les gens ne sont pas volontaires réellement. Estce qu'il y a une reconnaissance des faits? (raisonnement pragmatique) Oui, au début, elle a dit que c'était de la malchance et essayait de se justifier et finalement elle dit: "ben non j'avais bu ». Je pense que c'est bien de laisser le client exprimer justement les raisons pour lesquelles il est là. (raisonnement interactif) C'est facile pour moi de dire vous êtes ici parce que vous avez bu, mais j'attends que le client me le dise de lui-même. Sans reconnaissance, il n'y a pas de travail possible. (raisonnement procédural) Au début, j'attendais, mais finalement elle l'a dit qu'elle avait bu. Ce n'est pas facile pour un parent de venir admettre ses torts ce qui fait que ça peut être plus long, prendre des petits détours avant de l'admettre (raisonnement interactif) » (participant 1).

\section{Stratégies d’intervention utilisées par les intervenants}

Après la classification et le regroupement des données, cinq grandes catégories de stratégies d'intervention sont ressorties. Premièrement, les stratégies d'évaluation clinique contenaient à la fois les évaluations concernant la consommation de substances psychoactives (évaluer l'ampleur, les impacts et les raisons de la consommation) et l'évaluation des enjeux de la protection de la jeunesse (évaluer la reconnaissance des faits et les besoins de l'enfant et évaluer les capacités parentales). Deuxièmement, les stratégies relationnelles rassemblaient l'ensemble des stratégies où l'intervenant communique et écoute, évalue le réseau de soutien, essaie de gagner la confiance et de la renforcer, établit la relation d'aide, fait preuve de responsabilisation et essaie de susciter la réceptivité et la collaboration. Troisièmement, l'organisation des services regroupe l'ensemble des stratégies d'intervention qui abordait les services associés au programme Jessie et l'offre de services en général. Quatrièmement, on retrouvait les stratégies d'intervention qui touchent à la philosophie du programme Jessie (réduction des méfaits c. abstinence, volontarisme c. menace de placement) et à la philosophie du domaine de la protection de la jeunesse. Finalement, les stratégies d'intervention qui spécifiaient les modalités de la collaboration inter-établissements des services de protection de l'enfance et de la jeunesse et des services de réadaptation en dépendance ont été répertoriées. 
Les regroupements entre les types de raisonnement clinique et les stratégies d'intervention

Tableau 1. Stratégies d'intervention utilisées par les intervenants : le pourcentage d'extraits ( $n$ total $=684$ extraits) associés à chaque type de raisonnement clinique

\begin{tabular}{lrrrr}
\hline & Pragmatique & Procédural & Interactif & Conditionnel \\
\hline Stratégies d'évaluation clinique & $19,42 \%$ & $34,61 \%$ & $\mathbf{3 7 , 2 7} \%$ & $8,7 \%$ \\
Stratégies relationnelles & $33,14 \%$ & $20,38 \%$ & $\mathbf{4 2 , 0 6 \%}$ & $4,42 \%$ \\
Organisation des services & $\mathbf{6 9 , 9 1 \%}$ & $12,39 \%$ & $11,03 \%$ & $6,67 \%$ \\
Philosophie & $\mathbf{6 1 , 4 6 \%}$ & $23,43 \%$ & $15,11 \%$ & $0 \%$ \\
Collaboration inter-établissements & $\mathbf{8 6 , 6 8 \%}$ & $10,73 \%$ & $2,59 \%$ & $0 \%$ \\
\hline
\end{tabular}

Tel que présenté dans le tableau 1, les différents types de raisonnement clinique ont été utilisés pour chacune des stratégies d'intervention. Pour les stratégies d'évaluation clinique et relationnelles, on a noté une légère prédominance du raisonnement interactif, alors que pour les stratégies d'intervention que sont l'organisation des services, la philosophie et la collaboration inter-établissements, c'était plutôt le raisonnement pragmatique qui prédominait. Les stratégies d'intervention d'évaluation clinique et les stratégies relationnelles représentaient respectivement $45,18 \%$ et $44,15 \%$ des extraits répertoriés lors de l'entretien d'explicitation. Les regroupements entre ces stratégies d'intervention et les types de raisonnement clinique sont détaillés dans les tableaux 2 et 3 . Puisque l'organisation des services, la philosophie et la collaboration inter-établissements ressortaient peu des extraits répertoriés (respectivement 38,20 et 15 extraits), ils ne sont pas détaillés davantage.

Tableau 2. Stratégies d'évaluation clinique utilisées par les intervenants : le pourcentage d'extraits ( $n=309$ extraits) associés à chaque type de raisonnement clinique

Pragmatique Procédural Interactif Conditionnel

Évaluation consommation

de substances psychoactives

Évaluation ampleur

$13,97 \%$

$20,15 \%$

$42,5 \%$

$35,09 \%$

$8,44 \%$

Évaluation impacts

$6,55 \%$

$31,65 \%$

$42,59 \%$

$5,60 \%$

Évaluation raisons

$11,37 \%$

$55,52 \%$

$27,45 \%$

$10,48 \%$

Évaluation protection

de la jeunesse

$23,35 \%$

$46,50 \%$

$31,23 \%$

$10,89 \%$

Évaluation reconnaissance

des faits et besoins de l'enfant

$32,53 \%$

$28,26 \%$

$39,07 \%$

$9,32 \%$

Évaluation capacités parentales $16,29 \%$

$29,30 \%$

$33,18 \%$

$4,99 \%$

$27,47 \%$

$43,59 \%$

$12,65 \%$ 
En ce qui concerne les stratégies d'évaluation clinique spécifiques à la consommation de substances psychoactives, comme précisées dans le tableau 2, les intervenants ont fait davantage appel au raisonnement procédural. De manière détaillée, pour l'évaluation des impacts et des raisons associés à la consommation de substances psychoactives, les intervenants utilisaient surtout le raisonnement procédural tandis que lors de l'évaluation de l'ampleur de la consommation, ils avaient principalement recours au raisonnement interactif. Puis, lors de l'évaluation des enjeux associés à la protection de la jeunesse, le raisonnement clinique interactif était davantage utilisé.

Tableau 3. Stratégies relationnelles utilisées par les intervenants : le pourcentage d'extraits ( $n=302$ extraits) associés à chaque type de raisonnement clinique

\begin{tabular}{lrlll}
\hline & Pragmatique & Procédural & Interactif & Conditionnel \\
\hline Communication et écoute & $\mathbf{6 0 , 5 1} \%$ & $3,34 \%$ & $36,15 \%$ & $0 \%$ \\
Évaluation du réseau de soutien & $\mathbf{3 4 , 6 4} \%$ & $26,46 \%$ & $31,17 \%$ & $7,74 \%$ \\
Gagner et renforcer la confiance & $41,76 \%$ & $4,88 \%$ & $\mathbf{5 2 , 4 4} \%$ & $0,93 \%$ \\
Relation d'aide & $\mathbf{5 4 , 1 8} \%$ & $13,41 \%$ & $23,81 \%$ & $8,59 \%$ \\
Responsabilisation & $23,28 \%$ & $\mathbf{4 2 , 7 9} \%$ & $28,79 \%$ & $5,14 \%$ \\
$\begin{array}{l}\text { Susciter la réceptivité et la } \\
\text { collaboration }\end{array}$ & $21,65 \%$ & $25,45 \%$ & $\mathbf{4 7 , 8 7} \%$ & $5,04 \%$ \\
\hline
\end{tabular}

En ce qui a trait aux stratégies relationnelles (voir tableau 3), les types de raisonnement clinique associés étaient partagés. Ainsi, pour les stratégies de communication et d'écoute, d'évaluation du réseau de soutien et de la relation d'aide, le raisonnement pragmatique a été davantage utilisé. Pour la stratégie gagner et renforcer la confiance et susciter la réceptivité et la collaboration, le raisonnement interactif a été associé la moitié ou près de la moitié du temps. Enfin, la stratégie de responsabilisation était associée plus fréquemment au raisonnement procédural, un peu moins de la moitié du temps.

\section{Discussion et conclusion}

La présente étude visait à détailler le raisonnement clinique et les stratégies d'intervention des intervenants œuvrant auprès de parents négligents et consommateurs de substances psychoactives. Cette étude trouve sa pertinence dans la complexité du travail clinique réalisé par les intervenants du programme Jessie et autres programmes comparables. En effet, devoir concilier à la fois les nombreuses difficultés de ces parents, les divers enjeux d'intervention et les contraintes du processus judiciaire complexifie leur travail. Le contexte légal entraîne une gestion quotidienne du risque où l'intervenant doit prendre des décisions, et par le fait même des risques, tout en étant redevable devant la loi. Pour améliorer l'intervention et soutenir la pratique des intervenants, la présente étude avait pour but de revenir au cœur de l'acte professionnel, c'est-à-dire le raisonnement clinique et les stratégies d'intervention. 
L'utilisation de l'entretien d'explicitation a permis d'accéder au savoir tacite de ces intervenants, savoir difficilement accessible autrement (Martinez, 1997). Les analyses ont permis de révéler que l'ensemble des intervenants de l'étude ont utilisé, à un moment ou un autre, les différents types de raisonnement clinique, soit en ordre d'importance le raisonnement interactif, pragmatique, procédural et conditionnel. De plus, basé sur la typologie de Flemming (1991), il a été possible d'associer les différents types de raisonnement aux différentes stratégies d'intervention utilisées par les intervenants. L'étude étant réalisée auprès d'intervenants sociaux, il n'est pas surprenant que le raisonnement interactif, axé sur la relation, soit le raisonnement le plus utilisé lors de l'intervention. Un tel résultat est attendu considérant que les principes fondamentaux du programme Jessie sont la réduction des méfaits et l'approche motivationnelle, deux approches centrées sur les besoins du client (Comité de coordination du programme Jessie, 2014). II est clair que chez les intervenants rencontrés, ce type de raisonnement axé sur la relation, sur l'aspect émotif de l'évaluation, correspond à leur façon de travailler au quotidien. Plus spécifiquement, le raisonnement interactif est utilisé lorsqu'il est question des sujets délicats associés à la protection de l'enfant (évaluation de la reconnaissance des faits et des besoins de l'enfant, évaluation des capacités parentales), à l'ampleur de la consommation de substances, tout comme les stratégies d'intervention axées sur la confiance, la réceptivité et la collaboration.

En ce qui concerne la définition et la justification de leur évaluation, les intervenants utilisent le raisonnement pragmatique. Ce type de raisonnement et les actions qui en découlent guident l'entrevue vers le contexte du signalement, du programme et des ressources pour le parent. II n'est donc pas surprenant de voir associer le raisonnement pragmatique aux stratégies d'intervention qui touchent à l'organisation des services, aux caractéristiques associées à la philosophie du programme Jessie, à la philosophie de l'intervention en contexte de protection de l'enfance et de la jeunesse et aux modalités de collaboration entre les établissements participants.

Pour permettre le diagnostic clinique, le raisonnement procédural est mis de l'avant par les intervenants. Ce raisonnement vise à comprendre la situation familiale et à chercher des moyens et des options pouvant pallier les incapacités du parent. Il est utilisé par les intervenants, non seulement pour comprendre la situation, mais aussi pour déterminer les actions cliniques permettant un meilleur pronostic. Au cours de l'intervention avec un parent négligent et consommateur, on a observé ce raisonnement lors de l'utilisation des stratégies d'évaluation des impacts et des raisons associées à la consommation de substances psychoactives.

Enfin, le raisonnement conditionnel a été très peu utilisé par les intervenants participant à l'étude. Ce résultat provient sans doute du fait que ce type de raisonnement est habituellement présent plus tard dans le suivi (Flemming, 1991). Il est tout de même utilisé par les intervenants du programme Jessie pour faire des hypothèses quant à l'amélioration ou la dégradation ultérieure de la situation.

II importe de souligner que l'ensemble des raisonnements cliniques est rarement utilisé de façon isolée. En effet, comme mentionné par Unsworth (2004), les différents types de raisonnement sont souvent utilisés en alternance. Par le 
fait même, les différents types de raisonnement s'influencent mutuellement et sont complémentaires dans la prise de décisions des intervenants rencontrés. Chacun des raisonnements est utilisé en fonction des objectifs ciblés par les intervenants.

Si cette recherche explore un aspect peu étudié chez les intervenants œuvrant auprès de parents négligents et consommateurs, les résultats obtenus doivent être interprétés à la lumière de certaines limites. D'abord, bien que les vignettes cliniques aient été créées avec le souci de représenter des situations réalistes de familles référées au programme Jessie et que les comédiennes soient professionnelles et formées pour la situation clinique, certaines différences entre les comédiennes et la clientèle habituelle des parents négligents et consommateurs ont été notées (niveau de langage, apparence). De plus, les intervenants se sachant en simulation plutôt qu'en situation réelle ont pu modifier leur pratique habituelle. Bien qu'apprécié par les intervenants, l'entretien d'explicitation a exigé également un niveau d'exposition important et exigeant (c'est-à-dire être filmé, temps requis), ce qui a limité le nombre de participants. Avec un tel échantillon de convenance, il nous a été impossible d'effectuer des regroupements et des comparaisons selon des caractéristiques des intervenants de l'échantillon telles que le nombre d'années d'intervention ou le domaine d'étude. II est aussi probable que ces données ne se généralisent pas à l'ensemble des intervenants collaborant au programme Jessie, quoiqu'ils ne semblent pas être différents sur quelques informations sociodémographiques.

La présente étude a permis de mieux cerner la réalité de l'intervention auprès de parents négligents et consommateurs sans toutefois s'attarder à vérifier la justesse des stratégies d'intervention utilisées par les intervenants. Dans le futur, il serait intéressant de vérifier quelles stratégies d'intervention ou quels types de raisonnement clinique sont préférables selon les divers contextes rencontrés (par exemple présence des deux parents, substances consommées) ou selon les caractéristiques de l'intervenant (par exemple formation, expérience clinique). II faudrait également vérifier les types de raisonnement des intervenants selon leur milieu d'appartenance (protection de l'enfance et de la jeunesse c. réadaptation en dépendance). De même, il serait pertinent de mettre en perspective les présents résultats en considérant l'ensemble des facteurs (par exemple organisationnels, externes) potentiellement impliqués dans la prise de décisions des intervenants (Baumann et al., 2011).

En approfondissant les particularités de l'intervention auprès de cette clientèle, on serait à même de songer à une bonification des formations ou des supervisions cliniques destinées aux intervenants. En soi, il serait pertinent de vérifier l'utilité potentielle de l'entretien d'explicitation dans un contexte de formation continue, en raison de son potentiel pour l'amélioration et la consolidation de la pratique réflexive des intervenants (Guillemette, 2016). En effet, l'entretien d'explicitation permet d'engager activement l'intervenant dans l'explication du déroulement de son processus de raisonnement (suite des actions et informations considérées tout au long du raisonnement clinique) et de favoriser une prise de conscience du discours intérieur (Guillemette, 2016; Martinez, 1997). L'exposition au raisonnement clinique de différents intervenants, en opposition à un seul 
superviseur, représente, en effet, une riche occasion d'apprentissage professionnel (Andrews et Syeda, 2017).

En somme, cette étude représente l'une des rares études s'intéressant au raisonnement clinique d'intervenants œuvrant auprès de l'hétérogène population des parents négligents et consommateurs de substances psychoactives. L'utilisation de l'entretien d'explicitation a permis d'accéder au raisonnement clinique et aux stratégies d'intervention associées. L'identification des types de raisonnement clinique utilisés peut, ultimement, favoriser une prise de conscience du savoir implicite et ainsi, améliorer les pratiques (Carrier, Levasseur, Bédard et Desrosiers, 2012). En effet, la présente étude met en lumière quels raisonnements cliniques sont utilisés par les intervenants et comment se manifestent ces raisonnements dans les stratégies d'intervention mises de l'avant. Une telle connaissance est précieuse afin de favoriser une pérennité de l'expertise de ces intervenants, une pérennité nécessaire dans un contexte où le roulement du personnel est si important.

\section{Références}

American Psychiatric Association. (2013). Diagnostic and statistical manual of mental disorders DSM-5 (5e éd.). Arlington, VA: American Psychiatric Publishing.

Andrews, J. J. W. et M. M. Syeda. (2017). Clinical reasoning in school psychology: From assessment to intervention. Canadian Journal of School Psychology, 32(1), 3-15.

Barnard, M. (2007). Drug addiction and families. London, Angleterre: Jessica Kingsley Publishers.

Baumann, D. J., Dalgleish, L., Fluke, J. D. et Kern, H. (2011). The decision-making ecology. Washington, DC: American Humane Association. Repéré à https:// www.publicpolicy.com/upload/pdfs/ Juvenile\%20Justice\%20documents/ Race\%20Equity\%20Coalition/Decision_ Making_Ecology.pdf

Bourget, A. (2013). Explication du raisonnement clinique: méthodologie novatrice menant à l'identification de deux étapes de développement au cours des 12 mois entourant la fin de la formation préclinique et le début de la formation clinique d'étudiants en médecine. Recherches Qualitatives, 32(2), 1-26.
Carrier A., Levasseur, M., Bédard, D., et Desrosiers, J. (2012). Clinical reasoning process underlying choice of teaching strategies: A framework to improve occupational therapists' transfer skill interventions. Australian Occupational Therapy Journal, 59, 355-366.

Comité de coordination du programme Jessie. (2014). Programme Jessie: Une intervention concertée auprès de parents qui ont un problème de dépendance et de leurs enfants âgés de 0 à 5 ans, Guide de soutien à la pratique, édition révisée. Montréal, Québec: Centre jeunesse de Montréal - Institut universitaire et Centre de réadaptation en dépendance de Montréal - Institut universitaire.

Conners, N.A., Bradley, R.H., Mansell, L.W., Liu, J. Y., Roberts, T.J., Burgdorf, K. et Herrell, J.M. (2004). Children of mothers with serious substance abuse problems: An accumulation of risks. American Journal of Drug and Alcohol Abuse, 30(1), 85-100.

Deroche, H., Girard A., Naudin, D. et Bourget, A. (2016, 3-4 février). Exploration du raisonnement clinique des infirmiers anesthésistes diplômés d'état (IADE) en contexte de simulation : dévoilement de la face immergée de l'iceberg. Colloque international Décider en urgence. Acte du colloque. Paris (France). 
DiClemente, C.C. et Velasquez, M.M. (2002). Motivational interviewing and the stages of change. Dans W.R. Miller et S. Rollnick (dir.). Motivational interviewing: Preparing people for change. (p. 201216) Second edition. New York, NY: The Guilford Press.

Faucher, C. (2012). Développement d'un modèle de raisonnement clinique par son explicitation auprès d'optométristes de deux niveaux d'expertise contrastants. Recherches qualitatives, 31(2), 79-107.

Fleming, N.H. (1991). The therapist with three-track mind. American Journal of Occupational Therapy, 45(11), 10071014.

Fluke, J. D., Baumann, D. J., Dalgleish, L. I. et Kern, H. D. (2014). Decisions to protect children: A decision making ecology. Dans J. E. Korbin et R. D. Krugman (dir.). Handbook of child maltreatment (vol. 2) (p. 463-476). Dordrech, PaysBas: Springer.

Gallagher, F. (2014). La recherche descriptive interprétative: description des besoins psychosociaux de femmes à la suite d'un résultat anormal à la mammographie de dépistage du cancer du sein. Dans $M$. Corbière et $N$. Larivière (dir.). Méthodes qualitatives, quantitatives et mixtes: Dans la recherche en sciences humaines, sociales et de la santé ( $\mathrm{p}$. 5-27). Québec, Québec: Presses de I'Université du Québec.

Guillemette, F. (2016). Introduction: la pratique réflexive, tout le monde en parle, mais.... Approches inductives, 3(1), 1-6.

Hélie, S., Collin-Vézina, D., Trocmé, N., Turcotte, D. et Girouard, N. (2017). Étude d'incidence québécoise sur les signalements évalués en protection de la jeunesse en 2014 (ÉlQ-2014). Rapport final déposé à la Direction des jeunes et des familles du Ministère de la Santé et des services sociaux. Montréal: Centre intégré universitaire de santé et services sociaux-Centre sud de l'lle-de-Montréal. Repéré à http://centrejeunessedemontreal.qc.ca/
recherche/PDF/Publications/Rapport/ RapportFinal_EIQ2014.pdf

Higgs, J. et Jones, M.A. (2008). Clinical decision making and multiple problem spaces. Dans J. Higgs, M.A. Jones, S. Loftus et N. Christensen (dir.), Clinical reasoning in the health professions. Third edition (p. 3-17). Amsterdam, Pays-Bas: Elsevier.

Laventure, M., Couture, S., Fafard, F. et Pentecôte, C. (2016). Gestion du risque en maltraitance en contexte de réduction des méfaits. Validation préliminaire d'une grille d'observation des sphères de vie de parents consommateurs, (p. 78). Montréal: Centre intégré universitaire de santé et de services sociaux du CentreSud-de-l'Île-de-Montréal.

Martinez, C. (1997). L'entretien d'explicitation comme instrument de recueil de données. Expliciter, 20, 1-7.

Mayes, L. et Truman, S. (2002). Substance abuse and parenting. Dans M. Bornstein (dir.), Handbook of parenting: Social conditions and applied parenting ( $\mathrm{p}$. 329-359). Mahwah, New Jersey: Lawrence Erlbaum associates.

Miles, M.B. et Huberman, A.M. (2005). Analyse des données qualitatives. Bruxelles, Belgique : DeBoeck.

Nendaz, M. Charlin, B., Leblanc, V. et Bordage, G. (2005). Le raisonnement clinique : données issues de la recherche et implications pour l'enseignement. Pédagogie Médicale, 6(4), 235-254.

Newble, D., Norman, G. et van der Vleuten, C. (2000) Assessing clinical reasoning. Dans J. Higgs et M. Jones (dir.). Clinical Reasoning in the Health Professions, $2^{\text {nd }}$ édition. (p. 156-168) Oxford, Angleterre: Butterworth-Heinemann.

Richard, S. (2008). La délibération éthique chez les travailleuses et travailleurs sociaux en contexte d'intervention difficile: Quand le recours au "gros bon sens " et au raisonnement normatif est insuffisant pour interpréter la règle ou remettre en question la décision envisagée et l'action qui en découle. Reflets, 14(1), 200-217. 
Rix, G. et Biache, M.J. (2004). Enregistrement en perspective subjective située et entretien en re-situ subjectif: une méthodologie de la constitution de l'expérience. Intellectica, 38(1), 363396.

Ruest, M., Bourget, A., Delli-Colli, N., et Guay, M. (2017). Algo used by homecare non-occupational therapists selecting bathing assistive technology: Enhancing standardization by exploring clinical reasoning. Occupational Therapy in Health Care, 31(1), 20-33.

Schell, B.A. et Cervero, R.M. (1993). Clinical reasoning in occupational therapy: An integrative review. American Journal of Occupational Therapy, 47(7), 605-610.
Shlonsky, A. (2015). Current status and prospects for improving decision making research in child protection: $A$ commentary. Child abuse \& neglect, 49, 154-162.

Taylor, B.J. (2006). Factorial surveys: Using vignettes to study professional judgement. British Journal of Social Work, 36, 1187-1207.

Unsworth, C.A. (2004). Clinical reasoning: How do pragmatic reasoning, worldview and client-centredness fit? British Journal of Occupational Therapy, 67(1), 10-19.

Vermersch, P. (2006). L'entretien d'explicitation (5e éd.). Issy-les-Moulineaux, France: ESF éditeurs. 